\title{
Measurement of the hemoglobin oxygen saturation level with spectroscopic spectral-domain optical coherence tomography
}

\author{
Chih-Wei Lu, Cheng-Kuang Lee, Meng-Tsan Tsai, Yih-Ming Wang, and C. C. Yang* \\ Institute of Photonics and Optoelectronics, National Taiwan University, 1, Roosevelt Road, Section 4, Taipei, Taiwan \\ *Corresponding author: ccy@cc.ee.ntu.edu.tw
}

Received September 25, 2007; revised January 18, 2008; accepted January 19, 2008; posted January 25, 2008 (Doc. ID 87903); published February 19, 2008

\begin{abstract}
We report the measurement of the hemoglobin $(\mathrm{Hb})$ oxygen saturation level in human blood with a spectroscopic spectral-domain optical coherence tomography (SSD-OCT) system based on the crossover behavior of $\mathrm{Hb}$ and oxyhemoglobin $\left(\mathrm{HbO}_{2}\right)$ absorption coefficients around $800 \mathrm{~nm}$. By calculating the ratio of the exponential decay constant of A-mode scan signal in the long-wavelength range $(>800 \mathrm{~nm})$ over that in the short-wavelength range $(<800 \mathrm{~nm})$, the relative oxygen saturation level of $\mathrm{Hb}$ can be calibrated. Such a relative level can be used for practical diagnosis application after a golden standard is built by comparing the variation of the OCT result with that of a conventional method, such as the use of a blood gas analyzer. In our experiment, the variation curve based on the SSD-OCT measurement, which has the advantages of noninvasive, real-time, and high-resolution measurements, is coincident with that of using a commercial blood gas analyzer. (C) 2008 Optical Society of America
\end{abstract}

OCIS codes: $170.1650,180.1655$.

The hemoglobin $(\mathrm{Hb})$ oxygen saturation level is an important factor for many medical diagnoses, including obstructive sleep apnea [1] and transfusionrelated acute lung injury $[2,3]$. Some studies have led to the conclusion that cancerous tumors are related to the oxygenation level of blood [4]. Near cancerous cells, because of the extra need of oxygen supply and the extra blood vessels, tissue contains more oxyhemoglobin $\left(\mathrm{HbO}_{2}\right)$. An effective method for monitoring the $\mathrm{Hb}$ oxygen saturation level can help in cancerous tumor diagnosis. Several approaches have been used for monitoring the oxygenation level of blood, including the blood gas analysis for calibrating the oxygen pressure and optical measurement of $\mathrm{Hb}$ in extracorporeal blood circuits for calibrating the absorption spectrum [5]. However, they are either invasive or nonlocal measurements such that real-time and high-resolution diagnoses are difficult.

In this regard, optical coherence tomography (OCT) has the potential for providing us a noninvasive, real-time, and high-resolution tool for in vivo measurement of oxygenation level of blood. The use of the OCT technology for monitoring the oxygenation level of blood has been reported [6-8]. In one of the studies, the variation of the absorption spectrum was calibrated through the measurements of the reflection intensities from the top and bottom surfaces of a blood sample [6]. However, in an in vivo application, it is usually difficult to find clear boundaries for reflection measurement. An alternative approach was to use a nonlinear least-square fitting algorithm for analyzing the variation of absorption coefficient [7]. However, the spectral resolution based on the short-time Fourier-transform operation using a timedomain OCT system was not high. In the first in vivo OCT application to $\mathrm{HbO}_{2}$ measurement [8], Kagemann et al. directly measured the variation of blood absorption spectrum. Nevertheless, the useful spec- trum for analysis in this experiment was quite narrow, leading to a low sensitivity.

In this study, we demonstrate an alternative approach using the crossover behavior of the absorption spectra of $\mathrm{Hb}$ and $\mathrm{HbO}_{2}$ around $800 \mathrm{~nm}$ in wavelength (see Fig. 1 [9]). Based on this absorption behavior, we can utilize the concept of spectroscopic spectral-domain OCT (SSD-OCT) [10-12] to compare the A-mode scan profiles between the wavelength ranges shorter and longer than $800 \mathrm{~nm}$. With this approach, the calibration of the absolute absorption coefficient of blood sample is unnecessary and the diagnosis procedure becomes simpler. In this Letter, we report the implementation of an SSD-OCT system and the ex vivo measurement as well as the calibration results of different levels of $\mathrm{Hb}$ oxygen saturation in human blood. The ex vivo measurement represents the first and crucial step toward the use of the SSD-OCT technique for in vivo measurement and clinical diagnosis. In our experiment, the results based on the SSD-OCT measurement are consistent with those obtained from a blood gas analyzer.

In the SSD-OCT system, the femtosecond pulses with the central wavelength at $800 \mathrm{~nm}$ and the spec-

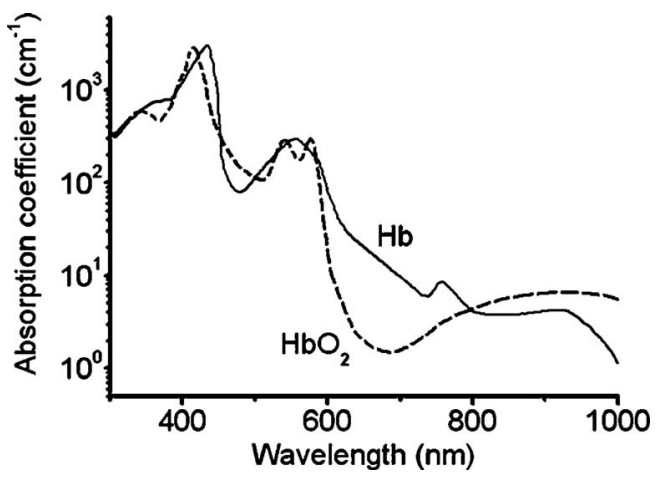

Fig. 1. Absorption spectra of $\mathrm{Hb}$ and $\mathrm{HbO}_{2}$ [9]. 


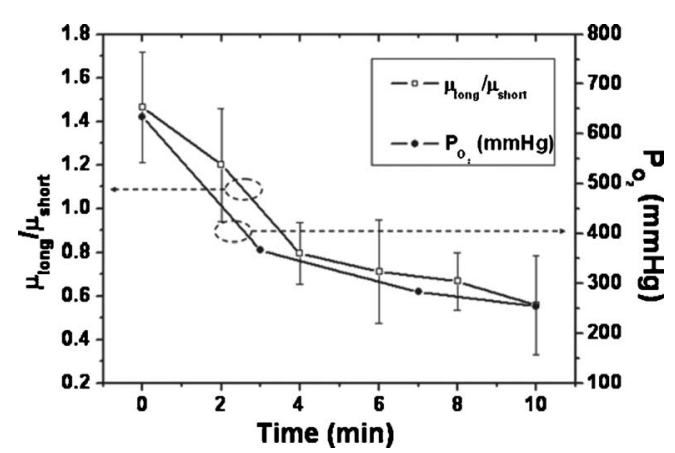

Fig. 2. Oxygen pressure level in the blood drop measured with a blood gas analyzer (circles with the right ordinate) and the ratio of $\mu_{\text {long }}$ over $\mu_{\text {short }}$ obtained from Figs. 3(a)-3(f) (squares with the left ordinate). The error bars for the ratio of $\mu_{\text {long }}$ over $\mu_{\text {short }}$ were obtained from the exponential fittings of ten sets of average A-mode scanning at various air exposure times.

tral full width at half-maximum at around $150 \mathrm{~nm}$ from a mode-locked Ti:sapphire laser $(150 \mathrm{MHz})$ are used as the broadband light source. The average output power of the laser is $380 \mathrm{~mW}$. The broadband signal is coupled into a free-space spectral-domain OCT system, in which the interfered signals are received by a spectrum meter including a grating with the spatial frequency at $600 / \mathrm{mm}$ (Thorlabs, GR50-0608) and a 2048-element CCD line scan camera (Basler, L104k). The interfered signals are then sent to a personal computer via a camera link by means of a highspeed frame grabber board (National Instruments PCI-1428). The standard procedures of spectraldomain OCT operation for image processing, including an inverse Fourier transform, are performed on the computer. After the interfered spectrum signals are acquired by the CCD, they are rescaled into the $k$ space. Then they are inverse Fourier transformed to give the spatial-domain image. Based on the concept of spectroscopic OCT, the short- $(<800 \mathrm{~nm})$ and longwavelength $(>800 \mathrm{~nm})$ bands are separated for inverse Fourier transform to obtain different A-mode scan results.

In the experiment, human blood from healthy volunteers was used as our sample. For anticoagulation, ethylenediamine tetraacetic acid was added to the collected blood. Before the SSD-OCT scan, a $10 \mathrm{ml}$ blood drop was placed on a culture dish. The dish was then placed in a sealed container filled with pure oxygen for $30 \mathrm{~min}$ to combine oxygen with $\mathrm{Hb}$. The period of $30 \mathrm{~min}$ is long enough for saturating the oxygen combination. After the blood drop is removed from the oxygen container for SSD-OCT scan in the air, the oxygen content decreases gradually with time. During the decay period of oxygen level, we also used a blood gas analyzer (TRUpoint) to monitor the oxygen pressure in the blood drop. The data of circles in Fig. 2 show the oxygen pressure measured with the blood gas analyzer as a function of air exposure period. The $\mathrm{p}_{\mathrm{O} 2}$ level in the blood drop becomes saturated after $10 \mathrm{~min}$ of air exposure.

With the sample preparation procedure described above, the human blood was scanned with the SSDOCT system. However, because a blood drop could be dried up under laser illumination during repeated OCT A-mode scanning, different blood drops were used for the OCT measurements of different air exposure periods. In this situation, different blood drops may have different concentrations of red blood cells. Figures 3(a)-3(f) show the A-mode scan profiles of the cases of $0,2,4,6,8$, and 10 min air exposure periods, respectively. Each profile in these figures was obtained by averaging $100 \mathrm{~A}$-mode scan results. In the spectral domain, an A-mode-scan profile contains 2048 pixels. They were divided into the shortand long-wavelength ranges separated at $800 \mathrm{~nm}$ for inverse Fourier transformation. The A-mode scan profiles in Fig. 3 show essentially exponential decay trends with their decay constants dominated by the absorption and scattering of red blood cells. The intensity decay profiles are fitted with exponential functions to give the decay constants $\mu_{\text {long }}$ (for the long-wavelength range) and $\mu_{\text {short }}$ (for the shortwavelength range). The fitting range starts from the depth of one-half the A-mode-scan maximum intensity and ends at the depth where the A-mode-scan intensity dropped to $10 \%$ of the maximum intensity. The full-spectrum A-mode scanning profile was used for determining the fitting range (the bottom portion of each part in Fig. 3). In each part of Fig. 3, the top and middle portions show the A-mode scan profiles and their fitting curves obtained by using the shortand long-wavelength spectral ranges, respectively. From the decay constants shown in Fig. 3, one can see that the OCT signals in the long-wavelength range decay faster than those in the shortwavelength range in the early stage [see Figs. 3(a) and $3(\mathrm{~b})]$. By assuming that the scattering of red blood cells is independent of their oxygenation condition, the trend in Fig. 3 indicates that the Hb absorption in the long-wavelength range is stronger when the oxygen content in blood is high. As oxygen content becomes lower, the absorption in the shortwavelength range becomes stronger than that in the long-wavelength range. Therefore, the decay constants in the short-wavelength range become larger than those in the long-wavelength range [see Figs. $3(\mathrm{c})-3(\mathrm{f})]$. It is noted that the decay constants in the

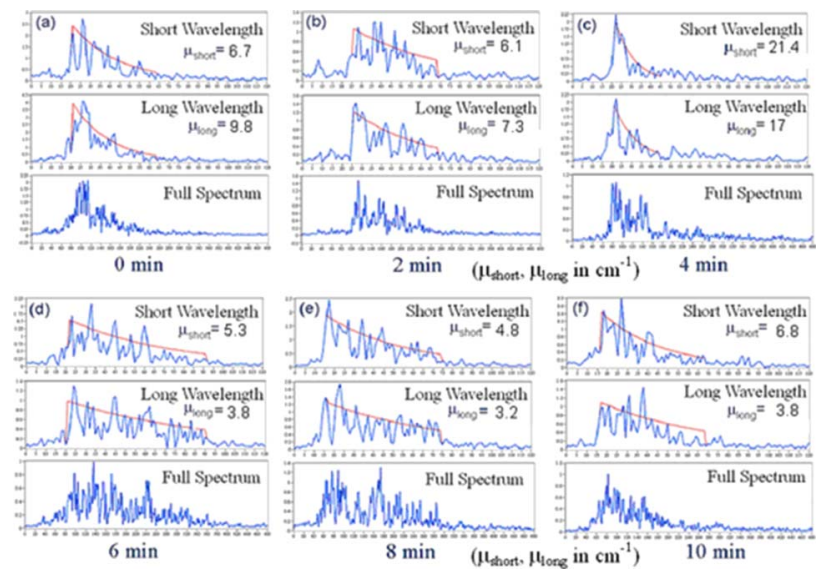

Fig. 3. (Color online) SSD-OCT A-mode scanning results in the cases of (a) 0, (b) 2, (c) 4, (d) 6, (e) 8, and (f) 10 min air exposure periods. The fitting curves are shown, and the calibrated decay constants are given. 
case of the 4 min air exposure period [Fig. 3(c)] are significantly higher than other cases, indicating that the red blood cell concentration in this blood drop is higher than others.

Different ratios of $\mathrm{Hb}$ over $\mathrm{HbO}_{2}$ content lead to different absorption spectra. When the $\mathrm{Hb}$ oxygen saturation level is high, absorption in the longwavelength range is higher than that in the shortwavelength range. Therefore, the ratio of the decay constant in the long-wavelength range $\left(\mu_{\text {long }}\right)$ over that in the short-wavelength range $\left(\mu_{\text {short }}\right)$ is related to the $\mathrm{Hb}$ oxygen saturation level. This ratio is weakly dependent on the red blood cell concentration in the blood drop sample. The data of squares in Fig. 2 show the results of $\mu_{\text {long }}$ divided by $\mu_{\text {short }}$ at different air exposure periods. The error bars were obtained from the exponential fittings of ten sets of average A-mode scanning at various air exposure times. One can see that the curve connecting the data points is quite consistent with the oxygen pressure variation obtained from the blood gas analyzer. The consistency implies that the demonstrated SSD-OCT measurement is a potentially useful approach for monitoring the $\mathrm{Hb}$ oxygen saturation level with the advantages of noninvasive, real-time, and highresolution measurements.

In summary, we have reported the measurement of hemoglobin oxygen saturation level of human blood with an SSD-OCT system based on the crossover behavior of hemoglobin and oxyhemoglobin absorption coefficients around $800 \mathrm{~nm}$. By calculating the ratio of the exponential decay constant of A-mode scan signal in the long-wavelength range over that in the short-wavelength range, the relative oxygen satura- tion level of hemoglobin could be calibrated. The variation curve based on the OCT measurement was consistent with that of using a commercial blood gas analyzer. This technique can be used for practical diagnosis application after a golden standard is built.

This research was supported by National Health Research Institute, Republic of China (grant NHRIEX96-9616EI).

\section{References}

1. L. D. Victor, Am. Fam. Physician 60, 2279 (1999).

2. M. A. Popvsky and N. R. Haley, Immunohematol 16, 157 (2000).

3. J. Bux, Vox Sang. 89, 1 (2005).

4. D. M. Brizel, S. P. Scully, J. M. Harrelson, L. J. Layfield, J. M. Bean, L. R. Prosnitz, and M. W. Dewhirst, Cancer Res. 56, 941 (1996).

5. I. Y. Petrova, R. O. Esenaliev, Y. Y. Petrov, H.-P. F. Brecht, C. H. Svensen, J. Olsson, D. J. Deyo, and D. S. Prough, Opt. Lett. 30, 1677 (2005).

6. D. J. Faber, E. G. Mik, M. C. G. Aalders, and T. G. van Leeuwen, Opt. Lett. 28, 1436 (2003).

7. D. J. Faber, E. G. Mik, M. C. G. Aalders, and T. G. van Leeuwen, Opt. Lett. 30, 1015 (2005).

8. L. Kagemann, G. Wollstein, M. Wojtkowski, H. Ishikawa, K. A. Townsend, M. L. Gabriele, V. J. Srinivasan, J. G. Fujimoto, and J. S. Schuman, J. Biomed. Opt. 12, 041212 (2007).

9. http://omlc.ogi.edu/spectra/index.html.

10. U. Morgner, W. Drexler, F. X. Kärtner, X. D. Li, C. Pitris, E. P. Ippen, and J. G. Fujimoto, Opt. Lett. 25, 111 (2000).

11. H. Cang, T. Sun, Z.-Y. Li, J. Chen, B. J. Wiley, Y. Xia, and X. Li, Opt. Lett. 30, 3048 (2005).

12. C. Xu, P. Carney, and S. Boppart, Opt. Express 13, 5450 (2005). 